Acta Horticulturae et Regiotecturae 2

Nitra, Slovaca Universitas Agriculturae Nitriae, 2019, pp. 80-83

\title{
MOVEMENT OF THE SUSPENDED FLOW IN THE OPEN IRRIGATION CHANNELS
}

\author{
Aybek ARIFJANOV ${ }^{1 *}$, Alisher FATXULLOEV ${ }^{\text {, Tatiana KALETOVÁ }}{ }^{2}$ \\ ${ }^{1}$ Tashkent Institute of Irrigation and Agricultural Mechanization Engineers, Uzbekistan \\ ${ }^{2}$ Slovak University of Agriculture in Nitra, Slovak Republic
}

\begin{abstract}
The article deals with the issues of turbulent movement of a suspended flow in open channels. Differential equations of a suspended flow were used with considering the interaction of the fluid and solid particles. The solution of equations for steady flow was obtained by a numerical method. Field data measured in irrigation channels in Tashkent and Parkent were used to estimate the velocity distribution over the depth of the stream. Developed method for calculating the formation of velocity field in statically and dynamically stable channels considered the turbulent characteristics and the presence of suspended sediments in the stream. The possibilities of practical application of the above-mentioned differential equations to the solution of the problem of a suspended solids flow's forming a velocity field were discussed. The developed analytical formulas make it possible to determine the value of flow parameters. The result is a new generalization of the mathematical model of the velocity field formation regularity at different flow saturations with a suspension. On its basis, the method is developed for calculating the parameters of the weighing process and transport of suspended particles in open channels.
\end{abstract}

Keywords: suspended solid flow, open channel, sediment, modelling

Flow turbulence affects the flow and channel cross section processes, determines the internal mechanism of the channel process, and forms the velocity structure of the suspended (two-phase) flow. What is the most important factor in assessing the stability condition of the channel? The importance and complexity of the turbulent motion of a suspended solid flow led to the development and improvement of various models of turbulent flow (Fidman, 1991; Frankl, 1953; Velikanov, 1955; Mikhailova, 1966).

There are numerous studies in open channels in this direction (e.g. Depeweg, 2002; Pal and Ghoshal, 2017; Lee et al., 2015). The most important kinematic and dynamic flow parameters, such as flow and channel interaction, velocity distribution and flow turbulence are studied. The result of the first approximation of mentioned parameters has been studied only, but the accuracy of predicting the formation of a stable channel section, considering transportation and sediment deposition, as a rule, remains low (Das, 2008; Karaushev, 1977). Therefore, many of the problems associated with the theory of interaction between the flow and the channel bed, as well as the formation of a stable cross section in the deformed channel, considering the turbulent structure of the suspended solid flow, are not studied thoroughly.

The difficulty and limitations in the practical implementation of existing models consist in the closure of the equations of motion system, as well as in considering that suspended particles affects the structure of the turbulent flow. In view of this, the streams with a low concentration of small particles are considered in some theories, which implies neglecting the effect of suspended sediment particles on the flow structure and the interaction of particles during their possible collisions. In this case, the averaged velocities of both phases are assumed to be the same, and when describing the velocity distribution profile, they use known dependencies derived for a single-phase flow in the beds, which significantly limits the scope of the solutions obtained.

\section{Material and methods}

In recent years, the theory of interpenetrating and interacting media, each phase of which is given by its equation (Rossinsky and Debolsky, 1960), has become applied widely in solving practical problems. In this theory, each medium is considered continuous; its movement is considered as a movement in a porous medium formed by the other phases. The system of equations developed according to this theory is closed, which makes it possible to theoretically investigate the phenomena occurring in a suspended turbulent flow.

When considering the movement of each phase separately, external forces are distributed to each phase. In addition, there is a force of interaction between the phases which, when considering a mixture in the flow, is an internal force. It follows that the forces acting on each phase consist of surface, mass and interaction forces. In this connection, the equations of motion for the $i$-th phase in differential form can be written as: 


$$
\rho_{1} \frac{d \bar{U}_{i}}{d t}=\operatorname{div} \bar{P}_{i}+\bar{\Pi}_{i}+\rho_{i} \bar{F}_{i}
$$

where:
$P_{i j}=-f_{i} \operatorname{grad} P+\frac{\partial \tau_{i j}}{\partial x_{i j}}-$ the stress tensor acting on the $i$-th phase

$\tau_{i j} \quad$ - internal stresses

$f_{i} \quad$ - volume content of the $i$-th phase $(i=1,2)$

The interaction force depending on the difference of the velocities of each phase is considered in the form:

$$
\Pi_{i}=\left(K \bar{U}-\overline{U_{s}}\right)
$$

Substituting the value of the parameters, equation (1) can be written in the form:

$$
\rho_{i}=\frac{d \bar{u}_{l}}{d t}=-f_{k} \operatorname{grad} P+\frac{d \tau_{k j}}{K\left(\bar{U}-\bar{U}_{s}\right)+\rho_{i} F_{i} d x_{j}}+
$$

where:

$\bar{U}, \bar{U}_{s}$ - the velocity vectors of the carrier fluid and solid particles, respectively

$K$ - coefficient of interaction between phases (carrier fluid and solid particles)

$f_{i}$ - mass forces, in this case, mainly the components of gravity are considered

The main and defining characteristics of the turbulent flow structure are turbulent shear stresses. Turbulent shear stresses are determined according to the model developed in (Borovkov, 1989):

$$
\tau_{i j l}=f_{i} \mu_{i}\left(-\frac{2}{3} \operatorname{div} \overline{V_{k}}+2 \frac{\partial v_{k j}}{\partial x_{j}}\right)-\int L V_{i} d_{n}
$$

with $j=l(\bar{n}=1,2,3)$

$$
\tau_{i j l}=f_{i} \mu_{i}\left(\frac{\partial v_{k l}}{\partial x_{j}}+\frac{\partial v_{k j}}{\partial x_{j}}\right)
$$

with $j \neq 1$

Then equation (3) considering (4) in projections on the coordinate axes $(j=x, y, z ; l=x, y, z)$ for the first phase of the suspended solid flow can be written as:

$$
\begin{aligned}
\rho_{1} \frac{d u_{1}}{d t}=\frac{\partial}{\partial x}\left(2 f_{1} \mu_{1} \frac{\partial u_{1}}{\partial x}\right)+ & \frac{\partial}{\partial y}\left(f_{1} \mu_{1}\left(\frac{\partial u_{1}}{\partial y}+\frac{\partial v_{1}}{\partial x}\right)\right)+\frac{\partial}{\partial z}\left(f_{1} \mu_{1}\left(\frac{\partial u_{1}}{\partial z}+\frac{\partial w_{1}}{\partial x}\right)\right) \\
& -L u_{1}+K\left(u_{1}-u_{2}\right)+\rho_{1} F_{x} \\
\rho_{1} \frac{d v_{1}}{d t}=\frac{\partial}{\partial y}\left(2 f_{1} \mu_{1} \frac{\partial v}{\partial y}\right)+ & \frac{\partial}{\partial x}\left(f_{1} \mu_{1}\left(\frac{\partial u_{1}}{\partial x}+\frac{\partial v_{1}}{\partial y}\right)\right)+\frac{\partial}{\partial z}\left(f_{1} \mu_{1}\left(\frac{\partial v_{1}}{\partial z}+\frac{\partial w_{1}}{\partial y}\right)\right) \\
& -L v_{1}+K\left(v_{1}-v_{2}\right)+\rho_{1} F_{y} \\
\rho_{1} \frac{d w_{1}}{d t}=\frac{\partial}{\partial z}\left(2 f_{1} \mu_{1} \frac{\partial w}{\partial z}\right) & +\frac{\partial}{\partial x}\left(f_{1} \mu_{1}\left(\frac{\partial w_{1}}{\partial x}+\frac{\partial u_{1}}{\partial z}\right)\right)+\frac{\partial}{\partial y}\left(f_{1} \mu_{1}\left(\frac{\partial v_{1}}{\partial z}+\frac{\partial w_{1}}{\partial y}\right)\right) \\
& -L w_{1}+K\left(w_{1}-w_{2}\right)+\rho_{1} F_{z}
\end{aligned}
$$

Respectively, for the second phase can be written as:

$$
\begin{aligned}
\rho_{2} \frac{d u_{2}}{d t}=\frac{\partial}{\partial x}\left(2 f_{2} \mu_{2} \frac{\partial u_{2}}{\partial x}\right) & +\frac{\partial}{\partial y}\left(f_{2} \mu_{2}\left(\frac{\partial u_{2}}{\partial y}+\frac{\partial v_{2}}{\partial x}\right)\right)+\frac{\partial}{\partial z}\left(f_{2} \mu_{2}\left(\frac{\partial u_{2}}{\partial z}+\frac{\partial w_{2}}{\partial x}\right)\right) \\
& -L u_{2}+K\left(u_{1}-u_{2}\right)+\rho_{2} F_{x} \\
\rho_{2} \frac{d v_{2}}{d t}=\frac{\partial}{\partial y}\left(2 f_{2} \mu_{2} \frac{\partial v}{\partial y}\right) & +\frac{\partial}{\partial x}\left(f_{2} \mu_{2}\left(\frac{\partial u_{2}}{\partial x}+\frac{\partial v_{2}}{\partial y}\right)\right)+\frac{\partial}{\partial z}\left(f_{2} \mu_{2}\left(\frac{\partial v_{2}}{\partial z}+\frac{\partial w_{2}}{\partial y}\right)\right) \\
& -L v_{2}+K\left(v_{1}-v_{2}\right)+\rho_{2} F_{y} \\
\rho_{2} \frac{d w_{2}}{d t}=\frac{\partial}{\partial z}\left(2 f_{2} \mu_{2} \frac{\partial w_{2}}{\partial z}\right) & +\frac{\partial}{\partial x}\left(f_{2} \mu_{2}\left(\frac{\partial w_{2}}{\partial x}+\frac{\partial u_{2}}{\partial z}\right)\right)+\frac{\partial}{\partial y}\left(f_{2} \mu_{2}\left(\frac{\partial v_{2}}{\partial z}+\frac{\partial w_{2}}{\partial y}\right)\right) \\
& -L w_{2}+K\left(w_{1}-w_{2}\right)+\rho_{2} F_{z}
\end{aligned}
$$

where:

$u, v, w \quad$ - components of velocity in projections on the coordinate axes

The field data were obtained in two irrigation channels in Tashkent and Parkent, northeastern part of Uzbekistan (Fig. 1). The channels are situated in SyrDarya River basin. The channels are modified with concrete $(n=0.0225)$, slope $0.14 \%$.

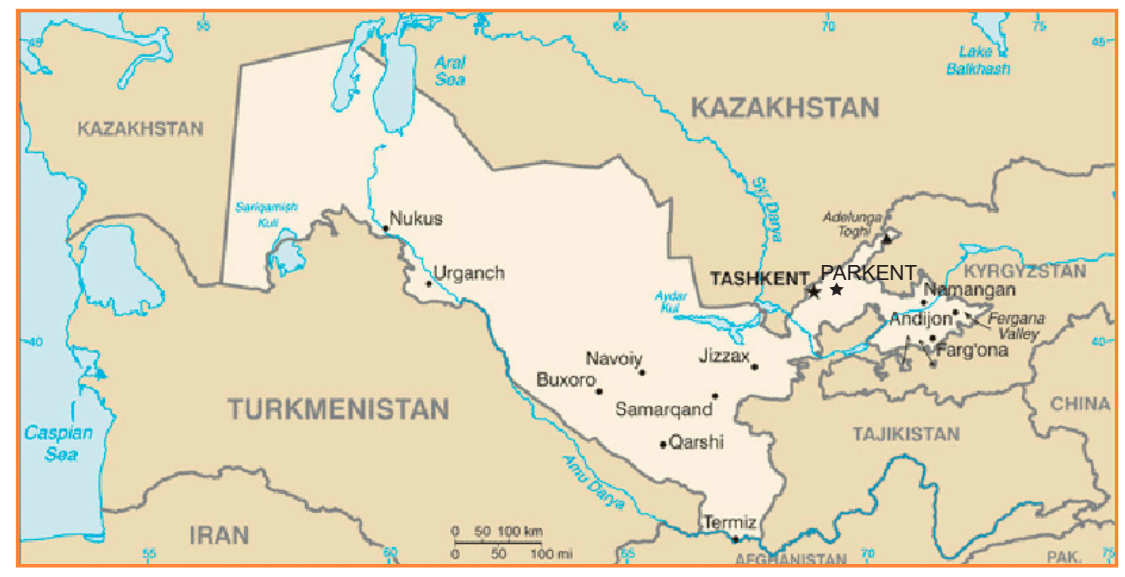

Figure 1 Map of Uzbekistan and location of field measurements Source: https://upload.wikimedia.org/wikipedia/commons/7/7e/Uzbekistan-ClA_ WFB_Map.png, modified by authors 


\section{Results and discussion}

A feature of the proposed system of differential equations is that the right side of the equations imply two additional terms $(L, L S)$, which characterize the turbulence of the channel flow-weighted. Solving a system of equations requires a search for flow parameters $(L, L s)$ characterizing the turbulence of a suspended solid flow, which is determined based on the developments given in Volgin (2009), Latipov and Umarov (1969), Latipov and Arifjanov (1994), Arifjanov and Fatkhullaev (2014).

To solve practical problems of the channel flow, it is necessary to develop a method for calculating the distribution of the averaging velocity of the suspended flow, which is used in wide variation ranges of the flow motion parameters conditions of the transported fluid and solid particles.

To develop a method for calculating the averaged velocity distribution over the depth of the suspended solid flow in the open channels, the system of differential equations (5 and 6 ) in the case of steady $\left(\frac{\partial u}{\partial t}=0\right)$, one-dimensional $\left(\frac{\partial u}{\partial x}=\frac{\partial u}{\partial z}=0\right)$ and uniform flow (assuming $f=s$ is the concentration of the second phase in the stream), the system of equations ( 5 and 6 ) is written in the form:

a) for carrier fluid:

$$
\frac{\partial}{\partial y}\left[(1-s) \mu \frac{\partial u}{\partial y}\right]+K\left(u_{s}-u\right)-(1-s) L u=-(1-s) \rho g i
$$

b) for suspended solids flow:

$$
\frac{\partial}{\partial y}\left[s \mu \frac{\partial u_{s}}{\partial y}\right]+K\left(u-u_{s}\right)-s L_{s} u_{s}=-s \rho_{T} g i
$$

where:

$\rho, \rho_{T}-$ density of the transported fluid and solid particles $i \quad$ - the slope of the water surface

The system of differential equations ( $7 a$ and $7 b$ ) is solved numerically using the finite difference method (Arifjanov

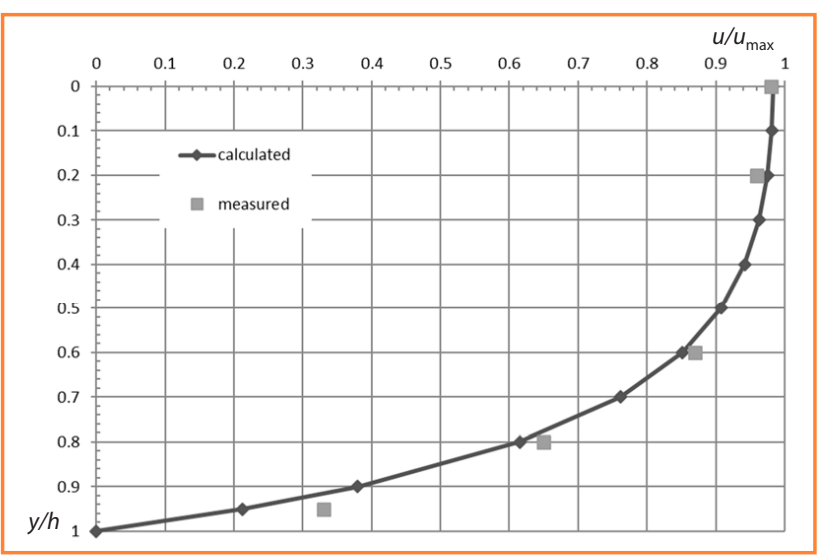

Figure 2 Comparison of calculated and measured data on the Tashkent channel

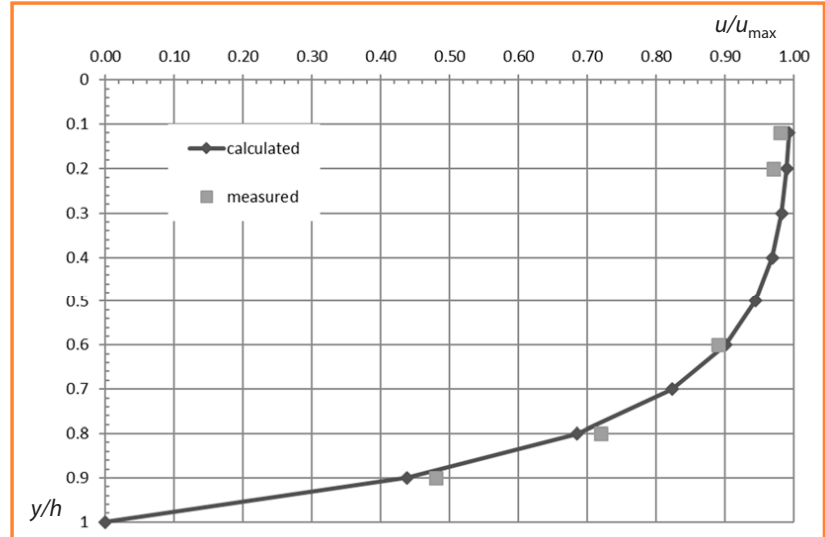

Figure 3 Comparison of calculated and measured data on the Parkent channel

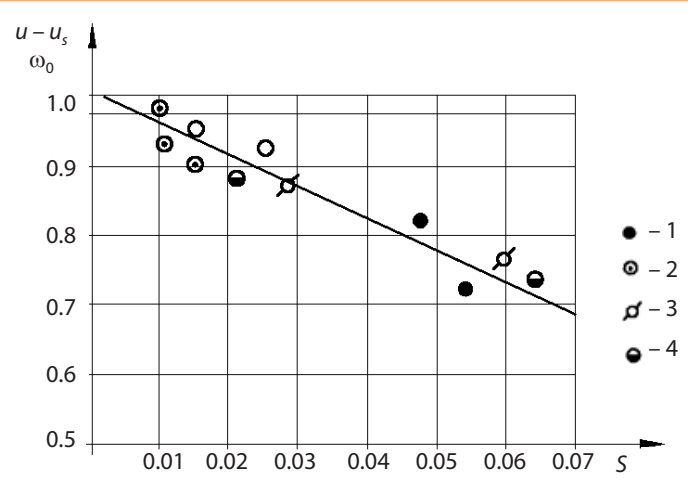

Figure 4 The dependence of the relative difference in the fluid velocity and particles on the concentration of suspension according to Grishanin (1992)

and Fatkhulloev, 2017). The field data obtained on Tashkent and Parkent channels, as well as data from other researchers were used to verify the reliability of the proposed dependencies on the calculation of the averaged velocity distribution over the depth of the flow for the existing computing methods comparative analysis. The comparison and comparative analysis with the results of field data and dependencies described in detail and given in Belolipetsky and Genova (2004), Borovkov et al. (2012), Debolsky et al. (1994), Ibad-Zade (1983), lovchu (2006) and Zidshlag (2008) were done.

The results of field studies were used to assess the formation of the velocity field according to equations ( $7 a$ and $7 \mathrm{~b}$ ). Those results of calculations present that the value of the relative velocity is the difference in the velocities of the transported fluid and the solid particle varies in depth with the flow (Fig. 2 and Fig. 3). This is obvious, since when the suspension flow moves in open channels, the concentration distribution of suspended particles through the flow depth is exponential. Therefore, the concentration of suspended particles increases with increasing depth. It is necessary to consider the fractional composition, which has a perceptible influence on the distribution of the relative velocity through the flow depth (Fig. 4).

Calculations show that the value of the relative longitudinal velocity is equal to the hydraulic extent only when are corrected for constraint. 
The dependence of the relative velocity on the saturation of the flow with a suspension is given in Fig. 4. The experimental data are given in this work. Particles increase with the concentration of sediment; the velocity of a solid particle lags far behind the flow, as can be seen in the Fig. 4. Note that the resulting relationship for determining the relative velocity takes place for a certain range of the size of solid sediment particles, which the flow can transport in a suspended state.

\section{Conclusion}

Based on the analysis and generalization, available theoretical and experimental studies in the field of dynamics of flows in channels improved mathematical model that describes the steady flow in open channels, considering the saturation of the flow sediment. As a result, it is possible to interconnect the formation of the channel and flow parameters in statically and dynamically stable sections of the channels.

The method for calculating the formation of the velocity field in statically and dynamically stable channels has been developed, considering turbulent characteristics and the presence of suspended sediments in the stream. The possibility of using the proposed methods for practical calculations is substantiated by data from the field studies on Tashkent and Parkent channels. The developed analytical formulas make it possible to determine the value of flow parameters. Whereby, comparison of the results provides satisfactory convergence of measured and calculated data.

\section{References}

ARIFJANOV, A.M. - FATKHULLAEV, A.M. 2014. Dynamics of a suspended flow in the channels. Tashkent : Fan, 2014, $180 \mathrm{p}$.

ARIFJANOV, A.M. - FATKHULLOEV, A.M. 2017. The program for calculation of dynamic parameters of a turbulent weighing flow. Copyright certificate for computer No. DGU 04730. Intellectual Property Agency of the Republic of Uzbekistan. Tashkent, 2017.

BELOLIPETSKY, V.M. - GENOVA, S.N. 2004. Computational algorithm for determining the dynamics of suspended sediment and bottom sediment in the river bed. In Computation. technology, vol. 9, 2004, no. 2, pp. 9-25.

BOROVKOV, V.S. - BAIKOV, V.N. - PISAREV, D.V. - VOLYNOV M.A. 2012. Local similarity of the flow and velocity distribution in turbulent flows. In Engineering - Construction Journal, 2012, no. 6, pp. 12-19. BOROVKOV, V.S. Channel processes and dynamics of river flows in urban areas. L: Gidrometeoizdat, 1989, 286 p.

DAS, A. 2008. Chance constrained design of trapezoidal channels. In Journal of Water Resources Planning and Management, vol. 134, 2008, pp. 310-313.
DEBOLSKY, V.K. et al. 1994. Dynamics of channel flows and lithodynamics in the coastal zone of the sea. Moscow: Nauka, 1994, $301 \mathrm{p}$.

DEPEWEG, H. 2002. Sediment transport applications in irrigation canals. In Irrigation and Drainage, vol. 51, 2002, pp. 167-179. doi: 10.1002/ird.49

FIDMAN, B.A. 1991. Turbulence of water flows. Leningrad : Hydrometeoizdat, 1991, $240 \mathrm{p}$.

FRANKL, F.I. 1953. On the theory of suspended sediment movement. Report. In USSR Academy of Sciences, vol. 92, 1953, no. 2, pp. 247-250.

GRISHANIN, K.V. 1992. Hydraulic resistance of natural channels. St. Petersburg: Gidromnteoizdat, 1992, 133 p.

IBAD-ZADE, Y.A. 1983. Transportation of water in open channels. Moscow : Stroyizdat, 1983, 555 p.

IOVCHU, Y.I. 2006. Model studies of rough channels of canals. Ways to improve the efficiency of irrigated agriculture: Sat. Art. FGNU "RosNIIPM"; by ed. VN Shchedrina - Novocherkassk : Helikon LLC, vol. 36, 2006, pp. 145-150.

KARAUSHEV, A.V. 1977. Theory and methods of calculation of river sediments. Leningrad : Gidrometeoizdat, 1977, 270 p.

LATIPOV, K.Sh. - ARIFJANOV, A.M. 1994. Issues of motion of a suspended flow in open channels. Tashkent: Publisher "Mekhnat", 1994, $110 \mathrm{p}$.

LATIPOV, K.Sh. - UMAROV, A.I. 1969. On the coefficient of interaction of multiphase media. In Questions of Mechanics, 1969, no. 7, pp. 57-60.

LEE, C-H. - HUANG, Z. - CHIEW, Y.-M. 2015. A multi-scale turbulent dispersion model for dilute flows with suspended sediment. In Advances in Water Resources, vol. 79, 2015, pp. 18-34. https://doi. org/10.1016/j.advwatres.2015.02.002

MIKHAILOVA, N. A. 1966. Transfer of solid particles by turbulent water flows. Hydrometeorological publishing house, 1966.

PAL, D. - GHOSHAL, K. 2017. Hydrodynamic interaction in suspended sediment distribution of open channel turbulent flow. In Applied Mathematical Modelling, vol. 49, 2017, pp. 630-646. doi: 10.1016/j.apm.2017.02.045

ROSSINSKY, K.I. - DEBOLSKY, V.K. 1960. River sediments. Moscow : Science, 1960, $215 \mathrm{p}$.

VELIKANOV, M.A. 1955. Dynamics of channel flows. Moscow : Gostekhizdat, 1955, 323 p.

VOLGIN,V.G. 2009. The influence of the length of the implementation of the velocity pulsations on the accuracy of the calculation of turbulent shear stresses. Moscow. In Vestnik MGSU, 2009, no. 9, pp. 93-99.

ZIDSHLAG, S. 2008. Modern method of measuring the flow rate used in the measurement of water consumption by the standard method. In Journal of Meteorology and Hydrology, 2008, no. 10, pp. 100-104. 